\title{
Faut-il délocaliser l'utopie ? A propos de la fonction prophétique de
} la Bible hébraïque

Thomas Romer

Citer ce document / Cite this document :

Romer Thomas. Faut-il délocaliser l'utopie ? A propos de la fonction prophétique de la Bible hébraïque. In: Autres Temps. Cahiers d'éthique sociale et politique. $N^{\circ} 51,1996$. pp. 45-51;

doi : https://doi.org/10.3406/chris.1996.1894

https://www.persee.fr/doc/chris_0753-2776_1996_num_51_1_1894

Fichier pdf généré le 26/03/2019 


\title{
Faut-il délocaliser l'utopie?
}

\section{A propos de la fonction prophétique de la Bible hébraïque}

\author{
Thomas Römer
}

On sait bien que les sociétés en crise engendrent toutes sortes d'utopies qui peuvent être collectives ou individuelles. L'intérêt de nos contemporains pour de «nouvelles formes » de religiosité qu'elles soient d'inspiration orientale ou américano-européenne apparaît dès lors comme la quête d'une utopie, d'un non-lieu, face à une société qui a perdu ses points de repères. Pourtant, ce déplacement idéologique ou spirituel ne suffit pas pour vivre le quotidien. D'où un intérêt grandissant pour l'éthique et la philosophie, disciplines qui apparaissent soudainement dans les émissions des chaînes de télévision commerciales. La crise se décline donc sur le double mode de l'aspiration utopique et de la nécessité d'affronter le présent de manière réaliste.

\section{L'Ancien Testament, une littérature de crise}

La Bible hébraïque, l'Ancien Testament, nous offre un paradigme pour comprendre la nécessité de maintenir le critique face à l'utopique qui, dans le langage des exégètes, se dit «eschatologie». L'Ancien Testament peut en effet être défini comme "une littérature de crise » dans le sens qu'une grande partie de son corpus s'est constitué à la suite de la grande catastrophe nationale que fut la destruction de Jérusalem et l'exil de sa population en Babylonie (597/87 avant notre ère). De nombreux courants idéologiques s'exprimant dans la Bible hé-

Thomas Römer est professeur d'Ancien Testament à l'Université de Lausanne (Suisse). 
braïque cherchent en effet à trouver des réponses face à cette crise. Certains insistent sur la sanction divine méritée (le courant deutéronomiste), d'autres accordent beaucoup d'importance aux médiations cultuelles (le courant sacerdotal), d'autres encore annoncent une nouvelle création, une ère de salut où le proton et l'eschaton se confondent (le courant eschatologique) : «Ne vous souvenez plus des premiers événements, ne ressassez plus les faits d'autrefois. Voici que moi je vais créer du neuf qui déjà bourgeonne ; ne le reconnaîtrez-vous pas ? " (Esaïe 43,19).

\section{Discours eschatologique et critique prophétique}

C'est à l'époque qui suivit l'exil que de nombreux livres prophétiques furent édités et augmentés d'oracles de salut annonçant aux destinataires un avenir de paix et de bonheur. Certains de ces oracles comportent sans doute une bonne dose d'utopie, mais, et cela est décisif, ils n'annulent pas la critique sociale et cultuelle des prophètes, notamment de ceux du VIII' ${ }^{\circ}$ siècle avant notre ère. Les éditeurs de l'Ancien Testament ont donc décidé de maintenir cette fonction prophétique de la Bible, et ceci dans un contexte où pourtant les discours apocalyptiques et utopiques faisaient davantage recette.

Ce n'était pas par intérêt antiquaire que les contestations prophétiques reçurent une place privilégiée dans le canon, mais parce que la mise à nu du dysfonctionnement de la société israélite du VIII ${ }^{\mathrm{e}}$ siècle avant notre ère pouvait aussi s'appliquer à l'époque perse. Celle-ci connut en effet des bouleversements économiques tout à fait comparables.

Le VIII' siècle avant notre ère, rappelons-le, est marqué par l'apparition d'une forme de proto-capitalisme (appelé souvent Rentenkapitalismus, capitalisme foncier) très répandue dans le Proche Orient ancien. La cour et l'aristocratie cherchent alors, par une comptabilité rigoureuse et un investissement réduit, à tirer le maximum de profit des terres affermées au détriment de ceux qui les cultivent. Ce système économique exprime la domination de la ville sur la campagne et transforme les paysans indépendants en serfs. Les paysans endettés sont obligés de se vendre eux-mêmes à leurs créanciers. La solidarité sociale et religieuse disparaît, laissant place à une logique de réussite économique qui creuse de plus en plus l'écart entre les riches et les pauvres.

Ce point est confirmé par l'archéologie. On trouve pour certains sites au VIII ${ }^{e}$ siècle deux types d'habitations : des édifices spacieux et élé- 
gants, formant un quartier à part et des habitations simples et précaires en périphérie, témoignant de la naissance d'un prolétariat urbain.

\section{La critique prophétique du VIII ${ }^{e}$ siècle avant notre ère}

C'est dans ce contexte que se situent les accusations d'Amos, d'Osée, d'Esaïe et de Michée, les premiers prophètes dont les oracles ont été édités. Les rédacteurs de leurs livres étaient peu friands d'anecdotes biographiques, ce qui leur importait c'était le message et non la personne. Michée et Amos étaient apparemment des campagnards judéens plutôt aisés, le dernier intervenant même dans le royaume d'Israël voisin. Esaïe est un aristocrate de la capitale judéenne. La seule information biographique éventuelle que nous possédons sur Osée est celle de son mariage avec une prostituée, mariage qui, dans le livre portant son nom, symbolise la relation entre Dieu et son peuple infidèle.

Malgré les nombreuses différences qui caractérisent ces figures prophétiques elles ont plusieurs dénominateurs communs, notamment la dénonciation d'une société qui a perdu sa cohérence sociale et idéologique et dont les dirigeants se comportent d'une manière arbitraire, ignorant l'ordre établi par Dieu. Selon l'idéologie royale commune au Proche-Orient ancien, Dieu a chargé le roi et ses ministres de garantir le maintien de cet ordre, en protégeant notamment les membres les plus faibles de la société. Or, ces prophètes observent que la réalité ne correspond pas à cette conception : "Qu'avez-vous à écraser mon peuple et à fouler au pied la dignité des pauvres » (Esaïe 3,15). La fustigation de cette dérégulation constitue un leitmotiv des livres prophétiques : «Ils disent bien au mal, et mal au bien. Ils posent les ténèbres comme lumière, et la lumière comme ténèbres " (Esaïe 5,20); «Écoutez donc, chefs de Jacob, magistrats de la maison d'Israël : N'est-ce pas à vous de connaître le droit ? Vous qui haïssez le bien et aimez le mal, qui arrachez la peau de dessus des gens et la chair de dessus de leurs os » (Michée 3,3). L'ordre établi par Dieu, son mishpat, assure l'harmonie et l'équilibre parmi les hommes, le non-respect de cet ordre a des conséquences fatales, puisque Dieu ne tolère pas l'injustice.

\section{L'exemple d'Amos}

Le message du prophète Amos peut se résumer en ces quelques mots : «La fin est venue ». Cette annonce qui parcourt son livre n'est pas à comprendre comme une pédagogie de la terreur, destinée à sus- 
citer auprès des destinataires une conversion de dernière minute. Il s'agit plutôt de la certitude prophétique que le mal qui caractérise la société de ses destinataires rend impossible toute perspective d'avenir. C'est à partir de cette «détonation » initiale qu'Amos va expliquer le pourquoi de la catastrophe imminente en analysant dans un esprit lucide et presque rationnel les injustices sociales : "Ils ont vendu le juste pour de l'argent et le pauvre pour une paire de sandales; ils sont avides de voir la poussière du sol sur la tête des indigents et ils détournent les ressources des humbles »(Amos 2,6-7). La partie centrale du livre d'Amos contient un long et impitoyable réquisitoire contre le royaume d'Israël. La critique vise une société qui ne sait plus distinguer le bien et le mal et dont même les institutions cultuelles, pourtant censées garantir la bienveillance divine, provoquent sa colère. Ce qui relie le présent au transcendant n'a aucune valeur, si le présent n'est pas marqué par la quête du droit et de la justice. Le religieux ne saurait servir d'échappatoire aux maux concrets de la société.

\section{La contestation du refuge cultuel}

«Je déteste, je méprise vos pèlerinages, je ne puis sentir vos rassemblements ... et dans vos offrandes rien qui me plaise; votre sacrifice de bêtes grasses, j'en détourne les yeux ; éloigne de moi le brouhaha de tes cantiques, le jeu de tes harpes, je ne veux pas l'entendre. Mais que le droit (mishpat) jaillisse comme les eaux et la justice (tsedaqah) comme un torrent intarissable! "(Amos 5,21-24, cf. également Esaïe 2,10-15). Le rédacteur du livre d'Amos ne donne pas de précision sur la situation originelle de cette diatribe, comme s'il s'agissait de la laisser hors contexte pour qu'elle interpelle directement chaque lecteur du texte. Le « Je» du prophète se confond ici avec le «Je» divin, un phénomène courant dans le discours prophétique. On remarque néanmoins l'absence de la formule du messager (« Ainsi parle Yhwh ») qui sert normalement à identifier le destinateur. Pourquoi cette absence ? Le prophète (ou son éditeur) avait-il peur de fonder directement dans l'autorité de Yhwh le discours très dur qu'il avait à communiquer ? En effet, ce message a quelque chose de blasphématoire. Dans les traditions du Pentateuque, c'est Dieu lui-même qui établit les règles de son culte, les sacrifices, etc.. Or, ici il est dit qu'il déteste ces pratiques. De plus, on trouve dans ces versets tout le vocabulaire technique de l'instruction sacerdotale (sentir favorablement, agréer, ...). Traditionnellement dans le culte, le porte-parole de la volonté de Yhwh n'était pas le prophète, mais le prêtre. En utilisant ce 
vocabulaire sacerdotal, Amos le transforme et en fait une parodie de la Torah sacerdotale. Le culte qui est ainsi rejeté n'est nullement un culte païen, mais bien un culte rendu au Dieu d'Israël. Ce qui est opposé à l'utopie d'un salut par le religieux est la nécessité d'une surabondance de droit et de justice, de mishpat et de tsedaqah.

\section{« Droit et justice »}

La signification de ces termes dépasse le seul cadre juridique. Il ne s'agit pas ici d'une allusion à un code juridique précis. Ces deux mots désignent un comportement d'éthique sociale et économique qui produit le shalom, c'est-à-dire une vie épanouie tant pour l'individu que pour la collectivité. Par l'exigence prophétique de mishpat et tsedaqah tous les autres lieux sont relativisés. L'absence de mishpat et de tsedaqah produisent une attitude religieuse narcissique coupée des réalités du présent (cf. l'insistance sur les adjectifs possessifs en Amos 5,21ss : vos fêtes, vos assemblées, vos offrandes).

\section{La fin des illusions}

L'absence de mishpat et de tsedaqah rend également caduc tout espoir d'un avenir meilleur. Ainsi, chez Amos, le «jour de Yhwh »qui désigne traditionnellement l'espérance en l'intervention salutaire de Dieu en faveur de son peuple - se transforme en un jour de jugement et de désastre: "Malheureux ceux qui misent sur le jour de Yhwh! A quoi bon? Que sera-t-il pour vous, le jour de Yhwh? Il sera ténèbres et non lumière " (Amos 5,18). Ici, le message prophétique marque une rupture. Le jour de Yhwh est devenu une menace radicale qui pèse désormais sur les destinataires d'Amos. Ils ne peuvent plus compter sur l'appui inconditionnel de leur Dieu, car pour défendre son droit qui est aussi le droit de l'homme, Yhwh est capable d'aller jusqu'à annihiler toute possibilité d'avenir.

En même temps que la négation du futur, Amos (ou peut-être un de ses rédacteurs) met en question l'événement fondateur, dans lequel Israël trouvait son identité, à savoir la sortie d'Égypte: «Pour moi, n'êtesvous pas comme des Nubiens, Israélites? - oracle de Yhwh. N'ai-je pas fait monter Israël du pays d'Égypte, les Philistins de Kaphtor (= Crète) et Aram de Qir? » (Amos 9,7). Israël est mis sur le même plan que les Philistins et les Araméens, ses ennemis traditionnels. Ceci revient à dire que la confession de foi en le Dieu de l'Exode ne comporte aucune garantie de salut si la réalité du peuple ne reflète cette libération. 


\section{La tension entre contestation prophétique et annonce de salut}

La violence du discours d'Amos a certainement scandalisé ses premiers auditeurs qui ont dû ressentir ses paroles comme blasphématoires. Et pourtant, elles ont été intégrées dans le canon des Écritures Saintes. Bien sûr, en lisant le livre d'Amos aujourd'hui on a l'impression que malgré la dureté de ses contestations tout se termine finalement par un happy end. A la fin du livre, le lecteur trouve l'annonce d'une restauration, la description d'une situation presque paradisiaque : «Voici que viennent des jours... où les montagnes font couler le moût et chaque colline ruisselle; je change la destinée d'Israël mon peuple... » (Amos 9,13-14). Cette finale constitue manifestement un ajout postérieur. «Des roses et de la lavande à la place du sang et du $f e u$ » observait le célèbre exégète Julius Wellhausen. Cet ajout présuppose la catastrophe du démantèlement du royaume de Juda par les Babyloniens. Il est dû aux derniers rédacteurs du livre qui voulaient redonner espoir à leur communauté après la catastrophe. Mais ceux qui ont ajouté au livre d'Amos cette perspective eschatologique n'ont pas essayé d'altérer les oracles de jugement précédents. L'épilogue du livre d'Amos, comme l'apocalypse de Jean à la fin du Nouveau Testament, expriment l'espoir que Dieu va intervenir pour les siens menacés par des puissances étrangères. Mais c'est seulement au dernier chapitre du livre que le lecteur peut accéder à un tel espoir. Autrement dit : les contestations et exigences qui précèdent ne sont nullement invalidées. Elles concernent chaque génération à nouveau et ne sont pas neutralisées par la finale du livre. Comme si les auteurs de cette finale étaient convaincus que l'attente du bonheur futur doit se conjuguer avec la dénonciation du mal présent.

\section{La fonction de la critique prophétique}

Les prophètes vétérotestamentaires n'étaient ni des réformistes ni des révolutionnaires et toutes tentatives d'en faire des sociauxdémocrates ou des communistes est anachronique et théologiquement inutile. Peut-être qu'Amos et Esaïe pourraient même être taxés d'hommes « de droite », dans le sens que leurs livres ne comportent aucune critique de l'institution monarchique elle-même. Les prophètes de la Bible hébraïque ne proposent aucun projet de société prêt à copier. Leur intérêt se situe sur un autre plan.

- Les prophètes perçoivent et dénoncent les perversions de la réalité historique. Le peuple qui à travers la tradition de l'Exode confesse un 
Dieu qui libère de l'esclavage, pratique lui-même l'exploitation des membres les plus faibles de la société.

- Les prophètes prennent position du côté de Dieu contre les structures du mal. Ils rappellent constamment que le bonheur ne peut être que le fruit de justice. Ce faisant, ils montrent où va une société qui bafoue la dignité de l'homme.

Mais avant tout, la présence des oracles prophétiques dans la Bible hébraïque est un rappel constant que le présent ne peut être négligé au détriment du futur, ou alors ce futur devient un futur de catastrophe et de jugement.

T. R.

\section{INDICATIONS BIBLIOGRAPHIQUES}

L. Monloubou, Les prophètes de l'Ancien Testament, Cahiers Évangile 43, Paris, Cerf, 1983.

T. Römer, "Amos : Les fondements de sa prophétie », Foi et Vie LXXXIII (Cahier biblique 23), 1984, pp. 26-34.

J. Blenkinsopp, Une histoire de la prophétie en Israël, Lectio Divina 152, Paris, Cerf, 1993.

\section{"Une religion qui n'a pas peur du siècle »}

Alain Duhamel

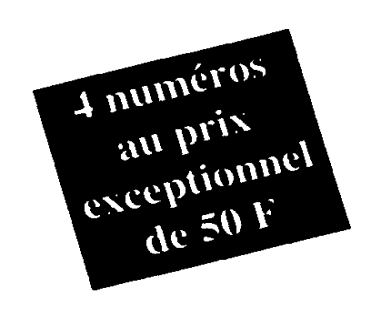

Pour aller plus loin dans la découverte du protestantisme

\section{Réforme}

Oui, je souhaite bénéficier de l'offre exceptionnelle de 4 numéros. $\mathrm{Ci}$-joint un chèque de $50 \mathrm{~F}$

Nom : Prénom :

Adresse complète :

Réforme, chaque semaine un regard protestant sur l'actualité 53-55, av. du Maine, 75014 Paris. Tél. 43203267 